\title{
QM/MM modelling of the TS-1 catalyst using HPCx
}

\author{
Judy To, ${ }^{a}$ Paul Sherwood, ${ }^{b}$ Alexey A. Sokol, ${ }^{a}$ Ian J. Bush, ${ }^{b}$ C. Richard A. Catlow, ${ }^{a}$ Huub J. J. van Dam, \\ Samuel A. French ${ }^{a}$ and Martyn F. Guest ${ }^{b}$
}

\author{
Received 23rd January 2006, Accepted 22nd March 2006 \\ First published as an Advance Article on the web 12th April 2006 \\ DOI: $10.1039 / b 601089 j$
}

\begin{abstract}
We report a series of computations on the active site in Ti-substituted zeolites, specifically TS- 1 . Hybrid QM/MM methods based on density functional calculations using the BB1K functional and a valence force field are used to study the processes of hydrolysis of Ti-O-Si linkages and inversion of the $\mathrm{TiO}_{4}$ tetrahedra. The structural features of the resulting series of tetra- and tripodal Ti moieties are in good agreement with data from EXAFS studies. The suggestion is made that the tripodal species will dominate in hydrous conditions, and that this is likely to be the chemically active form. We have made extensive use of the massively parallel HPCx computer system for these investigations and outline some of the technical developments to the ChemShell software that were needed to support the study.
\end{abstract}

\section{Introduction}

We present a study into the formation of active sites in $\mathrm{Ti}$ based zeolite catalysts. Titanium silicalite-1 (TS-1) is well known for its outstanding ability to catalyse various oxidation reactions with aqueous $\mathrm{H}_{2} \mathrm{O}_{2}$ as the primary oxidant under mild conditions. ${ }^{1,2}$ It is commonly believed that the isolated titanium atoms in the framework of TS-1 are the active sites for selective oxidation. However, the formation and structure of the active sites and the influence of water on the stability of the sites have not been definitively resolved. Recently, to address this problem, we investigated a number of possible structures of the active sites in TS-1, which could occur under dry and aqueous conditions. ${ }^{3}$ In particular, we identified the hydrolysis and inversion of $\mathrm{Ti}-\mathrm{O}-\mathrm{Si}$ bridges as the key mechanism, which helps to stabilise Ti within the framework of the zeolite. Here we validate and extend that study by applying a larger structural QM/MM model with a more extensive basis set and a more rigorous approach to the choice of the embedded QM cluster. We further report on the structure of the active sites and show it to be in good agreement with experiment.

In keeping with the theme of this special issue we will describe in some detail the way high-performance computing facilities have been used to address problems in materials science. The current project makes use of the ChemShell ${ }^{4}$ software, which provides quite general support for computational chemistry studies which require the integration of a number of different packages. The main target has been hybrid quantum mechanics/molecular mechanics (QM/MM) approaches, and we have developed methods within ChemShell to support such studies on a variety of chemical system types.

${ }^{a}$ Davy Faraday Research Laboratory, The Royal Institution of Great Britain, 21 Albemarle Street, London, UK W1S 4BS. E-mail: richard@ri.ac.uk; Fax: +44(0)20 7670 2958; Tel: +44 (0)2076702901

${ }^{b}$ CCLRC Daresbury Laboratory, Keckwick Lane, Daresbury,

Warrington, UKWA4 4AD. E-mail: p.sherwood@dl.ac.uk;

Fax: +44 1925 603634; Tel: +441925603553
This study described here uses the first such model, an approach to embedding within largely covalently bound crystalline networks such as aluminosilicates. Since the early work using this model, ${ }^{5}$ the code has been extended to handle biomolecular systems (using the CHARMM forcefield) and solid-state embedding into primarily ionic materials (using shell model potentials). ${ }^{6}$

ChemShell is modular in design, and the specific tools required to support $\mathrm{QM} / \mathrm{MM}$ studies (e.g. division of the atom lists, combination of forces and energies, support for link atoms, etc.) are provided in such a way that they can be used with a variety of QM and MM packages, in the current study we make use of GAMESS-UK ${ }^{7}$ and routines from the DL_POLY ${ }^{8,9}$ package, both maintained at Daresbury in support of the UK's Collaborative Computational Projects. Details of the specific forcefield choices and the way the codes are coupled are provided. Of particular interest in the current context is the possibility that ChemShell can be used on massively parallel computers. This is achieved by small modifications to the required packages (in this case GAMESS-UK) to allow them to be linked into ChemShell as a subroutine and the entire package executed in parallel. Some more information on the parallel implementation of ChemShell, and some of the more recent optimisations that are enabling large clusters to be studied, are outlined.

\section{The TS-1 catalyst system}

\section{Titanium in as-prepared dry catalyst}

TS-1 is based upon the silicalite framework (MFI) ${ }^{10}$ which contains a system of straight and sinusoidal intersecting channels of approximately $5.5 \AA$ in diameter that give rise to size and shape selectivity (Fig. 1).

The chemical properties of $\mathrm{TS}-1, \mathrm{Si}_{96-x} \mathrm{Ti}_{x} \mathrm{O}_{192}$ are controlled by the Ti ions, up to $x \approx 2.5$, substituted into the silicalite framework. ${ }^{11}$ Since a low concentration of Ti ions is introduced into the framework, it is generally believed that the catalytically active $\mathrm{Ti}$ ions are site isolated from one another. 


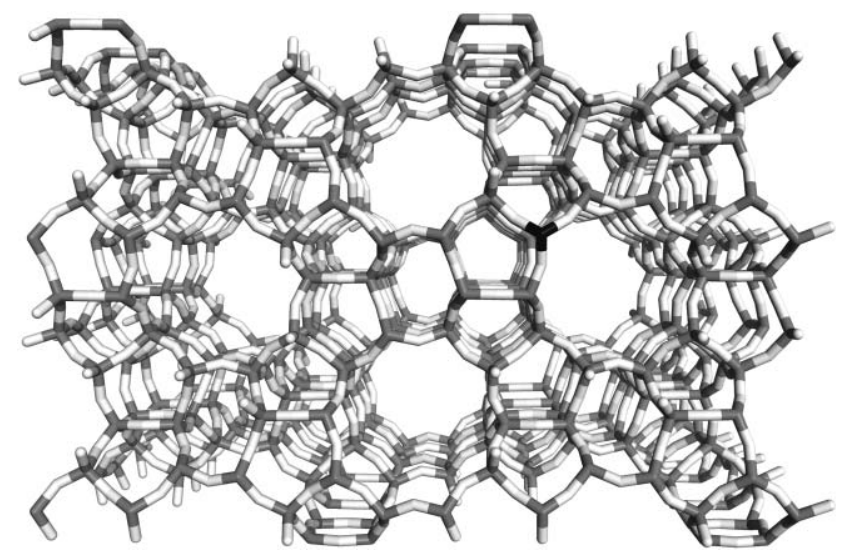

Fig. 1 Structure of TS-1, Si (grey) and Ti (black) tetrahedra connected via oxygen bridges (white).

Ti substituted zeolites in anhydrous conditions are characterised by:

- XRD measurements, which demonstrate that the unit cell parameters of silicalite expand linearly with $\mathrm{Ti}$ content when $\mathrm{Ti}$ atoms are inserted into the framework. ${ }^{12}$

- The UV-vis spectra revealing a well-defined absorption band at $\sim 48000 \mathrm{~cm}^{-1}$, which is absent from silicalite. This signal is characteristic of tetrahedral $\mathrm{Ti}$ and corresponds to a charge transfer from the oxygen ligand to the Ti centre in the $\mathrm{TiO}_{4}$ unit. $^{11,13}$

- The absence of an UV-vis absorption band at $30000 \mathrm{~cm}^{-1}$, which is characteristic of octahedral extra-framework Ti ions $\left(\mathrm{TiO}_{2}\right){ }^{11}$

- The XANES spectrum recording a narrow peak at $4967 \mathrm{eV}$, which can only be explained in terms of the Laporte allowed $\mathrm{A}_{1} \rightarrow \mathrm{E}$ characteristic transition of the $(1 \mathrm{~s})^{2} \rightarrow(1 \mathrm{~s})^{1}(3 \mathrm{~d})^{1}$ excitation in tetrahedral $\left[\mathrm{TiO}_{4}\right]$ structures. $^{14}$

- The Ti(2p) XPS peak at $459.8 \mathrm{eV}$, which has been assigned to tetrahedral framework $\mathrm{Ti}^{2}$

- The absence of the XPS peak at $458.3 \mathrm{eV}$, which is indicative of extra-framework Ti species. ${ }^{2}$

- Both infra-red and Raman spectra recording a band at $960 \mathrm{~cm}^{-1}$ (absent in silicalite), ${ }^{15}$ which has been attributed to the stretching vibration of the $\mathrm{Si}-\mathrm{O}$ bond in $\mathrm{Si}-\mathrm{O}-\mathrm{Ti}$ bridges, or more recently to the $\mathrm{Ti}-\mathrm{O}-\mathrm{Si}$ stretch. ${ }^{16}$

It should be noted that the interpretation of the IR band in the last point has been a matter of debate. ${ }^{17}$ Originally, it was first attributed to the $\mathrm{Si}-\mathrm{O}$ stretching vibration, but later it was found that titanyl $(\mathrm{Ti}=\mathrm{O})$ and silanol $(\mathrm{Si}-\mathrm{OH})$ groups also resonate in the same frequency region. ${ }^{15}$ Emission excitation spectra indicate that, contrary to previous assertions, the $960 \mathrm{~cm}^{-1}$ band corresponds to the Ti-O-Si stretching mode in TS-1. ${ }^{16}$

\section{The rôle of water and structure of active sites}

Both theory and experiment agree that the titanium centres in TS-1 are tetravalent and four-coordinate in anhydrous conditions. However, experiment shows that when exposed to adsorbates such as water, the $\mathrm{Ti}$ increases its coordination number reversibly from four to five or six: $:^{2,18}$
- The absorption band in the UV-vis spectrum, which decreases in energy from $48000-50000 \mathrm{~cm}^{-1}$ in the dehydrated form to $42000 \mathrm{~cm}^{-1}$ on addition of $\mathrm{H}_{2} \mathrm{O}$. This band is assigned to charge-transfer transitions between the new ligand and $\mathrm{Ti}$ ions and is consistent with an increase in the $\mathrm{Ti}$ coordination shell. ${ }^{19}$

- The IR band shifts from $960 \mathrm{~cm}^{-1}$ under anhydrous conditions to $975 \mathrm{~cm}^{-1}$ on addition of $\mathrm{H}_{2} \mathrm{O} \cdot{ }^{20}$

- EXAFS studies demonstrate that the Ti-O-Si distances of the four framework bonds undergo a stretch of $0.02 \AA$ upon adsorption of $\mathrm{H}_{2} \mathrm{O} .^{21}$

- XANES data indicate a change in the local geometry from a tetrahedral to octahedral type similar to that observed in anatase. $^{20}$

Thus, the ability of $\mathrm{Ti}$ to change its coordination number between four and six is widely accepted, but how this occurs in the zeolite framework is not fully understood. It has been reported that the coordination expansion takes place with the hydrolysis of the Ti-O-Si bridges forming Ti-OH and $\mathrm{Si}-\mathrm{OH}$ groups. ${ }^{17}$ In fact, experimental data from IR and ${ }^{17} \mathrm{O}$ MAS NMR of TS-1 treated with isotope-labelled water have confirmed the formation of Ti-OH in hydrated TS- $1 .^{22}$ Furthermore, Lamberti et al. reported that even for samples dehydrated in a carefully controlled atmosphere at $400 \mathrm{~K}$, a considerable fraction of sites exhibit the substitution of a bridged oxygen with two $\mathrm{OH}$ groups. ${ }^{11}$ This observation is further supported by a parallel photoluminescence study, where the presence, in both emission and excitation spectra, of two distinct bands clearly indicates two structurally different Ti sites. ${ }^{23}$

Tri-, bi-, and monopodal structures have been suggested as representative of the active sites in (an)hydrous titanosilicates, arising from the progressive hydrolysis of a tetrapodal T-site. The terms tetra-, tri-, bi-, and monopodal indicate four, three, two and one Ti-O-Si bridges anchoring the metal to the silica framework, respectively (Fig. 2). An alternative bipodal titanyl
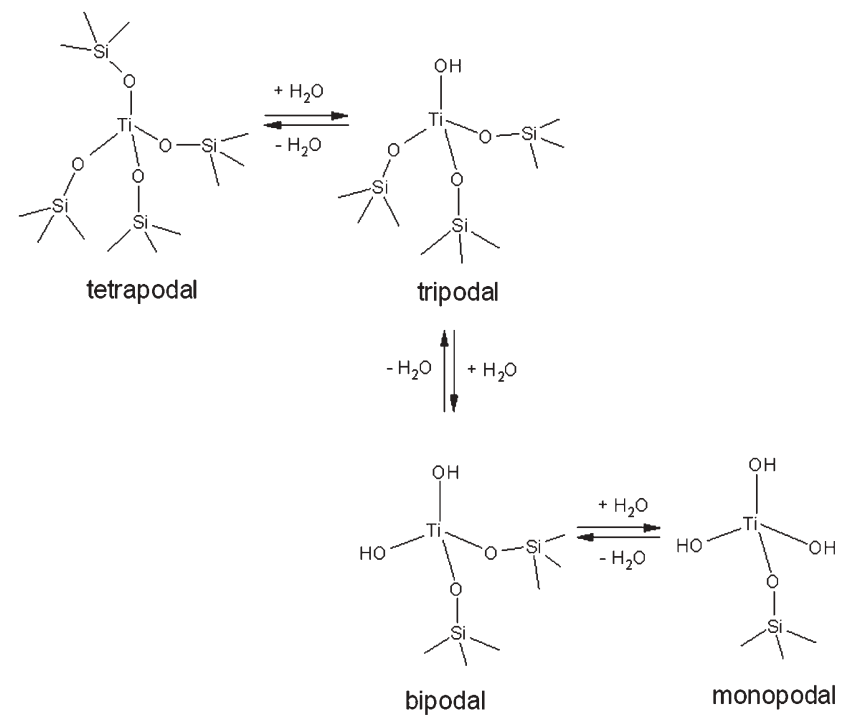

Fig. 2 Proposed models of tetrahedral $\mathrm{Ti}$ sites in (an)hydrous titanosilicalites. $^{29}$ 
model with a dangling $\mathrm{T} i=\mathrm{O}$ group has also been advanced in the literature ${ }^{24}$ but did not find experimental support.

It is generally not possible to differentiate experimentally the proposed models (except for the titanyl model since $\mathrm{Ti}=\mathrm{O}$ should be about $0.1 \AA$ shorter than a Ti-O bond to a tetrahedrally coordinated Ti). For example, in an attempt to distinguish between tetrapodal and tripodal environments Gleeson et al. performed a more detailed EXAFS investigation on a series of TS- 1 catalysts with different Ti contents, ${ }^{25}$ where both tetra- and tripodal structural models were used to simulate the EXAFS spectra with similar success.

The early computational work on tetrahedral Ti centres was based on generic clusters, and therefore there was no real distinction of the active sites between micro- and mesoporous silicas. ${ }^{26}$ In particular, Sinclair et al. proposed that the actual catalytic site in Ti-MCM-41 is the tripodal complex and that an appreciable concentration of bipodal complexes would also be present. Several theoretical cluster studies investigated the adsorption of water and found that the expansion of the tetrahedral $\mathrm{Ti}$ sites to a coordination of five or six is an energetically favourable process. ${ }^{26-28}$ To account for framework constraint effects in a crystalline environment, ZicovichWilson et al. performed periodic Hartree-Fock calculations on $\mathrm{H}_{2} \mathrm{O}$ physisorption (hydration) and hydrolysis on Ti-chabazite, the unit cell of which is significantly smaller than that of Ti-silicalite. ${ }^{29}$ Upon addition of $\mathrm{H}_{2} \mathrm{O}$, physisorption was found to be more energetically favoured than hydrolysis, however, the activation barrier of about $84 \mathrm{~kJ} \mathrm{~mol}^{-1}$ for hydrolysis indicated that the reaction is kinetically possible at usual working temperatures, as for instance, during the calcination of the samples. Similarly, QM/MM calculations by Ricchiardi et al. found that insertion of one or two $\mathrm{H}_{2} \mathrm{O}$ molecules into the coordination sphere of $\mathrm{Ti}$ is energetically favourable, whereas the hydrolysis of one Ti-O-Si bridge is an endothermic process. ${ }^{30}$ These calculations suggested that when the $\mathrm{Ti}-\mathrm{O}-\mathrm{Si}$ or $\mathrm{Si}-\mathrm{O}-\mathrm{Si}$ bridges are broken, the rearrangement of the local structure is not favoured by framework constraints and therefore more accessible Ti sites are not formed. More recently, Damin et al. studied the interaction of one $\mathrm{H}_{2} \mathrm{O}$ with Ti-zeolites, adopting both finite clusters and embedded clusters as models of the Ti site. ${ }^{31}$ The authors reported a substantial enhancement in the binding energy of water, rising from $2.1 \mathrm{~kJ} \mathrm{~mol}^{-1}$ for the unconstrained $\mathrm{Ti}\left(\mathrm{OSiH}_{3}\right)$ cluster to $16.9 \mathrm{~kJ} \mathrm{~mol}^{-1}$ for the embedded $\mathrm{TiSi}_{17} \mathrm{O}_{26} \mathrm{H}_{20}$ cluster. These results highlight the important role played by the zeolitic framework constraints in enhancing the reactivity of Ti centres toward $\mathrm{H}_{2} \mathrm{O}$ molecules. More recently, there also appeared a number of studies of the hydration of tetrapodal Ti sites (see for example ref. 32).

We have undertaken a series of calculations on the proposed models aiming to identify the lowest-energy structures, thus pinning down the actual active sites in the catalysts, but also providing starting models for refinement of experimental data. The work is focused on the effect that hydrolysis has on the geometry, coordination and stability of the Ti sites.

The highlight of this work is the proposal of a new model of the tetravalent $\mathrm{Ti}$ site, which is based on the hydrolysis and inversion of tetrahedral sites in the zeolite. The characteristic features of this model are closely related to a number of structures in silica. For example, it has been postulated that the hydrolysis step during silica synthesis occurs through an $\mathrm{S}_{\mathrm{N}} 2$ mechanism with inversion of a silicon tetrahedron. ${ }^{33} \mathrm{~A}$ similar mechanism was reported for positively charged oxygen vacancy defects ( $E^{\prime}$ centres) in $\alpha$-quartz and amorphous silica. ${ }^{34,35}$ It was suggested that the $E^{\prime}$ centre undergoes a distortion to a stable puckered configuration, which is accompanied by a large relaxation of the $\mathrm{Si}$ atoms adjacent to the oxygen vacancy. Recently, Sokol et al. ${ }^{36,37}$ advanced the hydrolysis and inversion model for the stabilisation of intrinsic defects, e.g. vicinal disilanols and trigonal Al species, in hydrated siliceous and aluminosilicate zeolites.

\section{Computational approach}

In this section we outline some aspects of the models implemented in ChemShell ${ }^{5,6}$ and their implementation on HPCX.

\section{QM/MM methodology}

GAMESS-UK was used for the calculation of the energy and gradient of the QM parts, and for the MM part we used the internal MM functionality of ChemShell, which is constructed using routines from the DL_POLY package ${ }^{8,9}$ with additional code added to deal with the zeolite forcefield. The QM/MM model is of the electrostatic embedding type (model B in the classification of Bakowies and Thiel ${ }^{38}$ ), which means that the electrostatic potential from the classical part of the system is included in the 1-electron Hamiltonian of the QM calculation. The calculations make use of finite MM clusters, rather than performing calculations of the infinitely repeating periodic system. This is partly for reasons of computational convenience, as it avoids the need to introduce code for Ewald summation into the QM/MM coupling calculation. However, the approach also has the desirable characteristic that we are modelling a very dilute defect, in which the active sites do not interact, and this is usually an appropriate model for real catalytic systems. Electrostatic embedding approaches which involve QM and MM regions connected by bonds necessarily require careful treatment of the interface region. This issue has been discussed in some detail, ${ }^{6}$ and a number of treatments are in use. The current model involves the termination of the QM region by capping hydrogen atoms, forming terminal $\mathrm{Si}-\mathrm{H}$ and $\mathrm{O}-\mathrm{H}$ groups. The boundary region is showed schematically in Fig. 3, Q and M label quantum and molecular mechanically modelled atoms respectively, and the numbers denote the number of bonds distant from the QM/MM boundary.

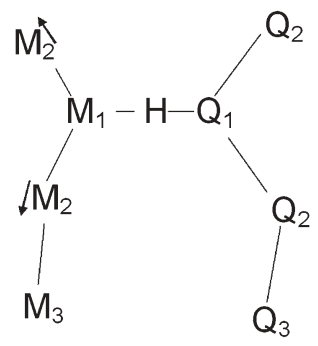

Fig. 3 Charge shift termination of the QM region. 
The first step in building the QM/MM model is to remove classical charges from the centres which are to be treated quantum mechanically, ensuring that the total charge removed from the system is the same as the charge of the QM cluster that is going to be used, usually this is zero for simple clusters comprising $\mathrm{Si}$ or $\mathrm{Ti}$ at the $\mathrm{T}$-sites. To ensure this neutrality the charges on the $\mathrm{M}_{1}$ centres are also reduced by $-0.5 \times$ the oxygen charge for each cross-interface bond. 5

In our zeolite QM/MM model we then delete the classical charge on the $\mathrm{M}_{1}$ centre, and adjust the charges on any directly connected classical centres $\left(\mathrm{M}_{2}\right)$ to preserve the charge on the classical system, a compensating dipole is added to the $\mathbf{M}_{2}$ centres. We refer to this as the "charge shift" termination scheme. $^{5}$

\section{The parallel implementation of ChemShell}

The parallel implementation of ChemShell is complicated by the fact that its main role is to interface other packages; the strategy must enable the parallelisation of these packages to be exploited wherever possible while retaining, as far as possible, the modularity of the overall ChemShell package. The approach taken is to consider the interfaced packages in groups, the first are built into the binary and follow the SPMD parallelisation strategy, in which the same code executes on each node, the second group are executed as stand-alone programs, with communication occurring through files. The first approach is used when interfacing GAMESS-UK (also DL_POLY, GULP and NWChem) on a massively parallel system, and is described briefly here.

At the centre of ChemShell lies a $\mathrm{Tcl}^{39}$ interpreter and no attempt has been made to parallelise this. The standard Tcl source code is used, and it is executed only on one processor, that is processor 0 . After the initial handshaking required by the message passing software, the remaining processors then enter a message passing receive call, at which point they block until a message (originating from processor 0 ) causes them to either pass control to a parallel subroutine or to exit. The available options are set up at the start of the run, each package interfaced to ChemShell may register the parallel entry point using a $\mathrm{C}$ function. As an example, consider the GAMESS-UK interface. Here gamess_wrap() is a small C function which calls the main program of GAMESS-UK, and the parallel code is compiled using -DMASTERSLAVE to activate the illustrated parts:

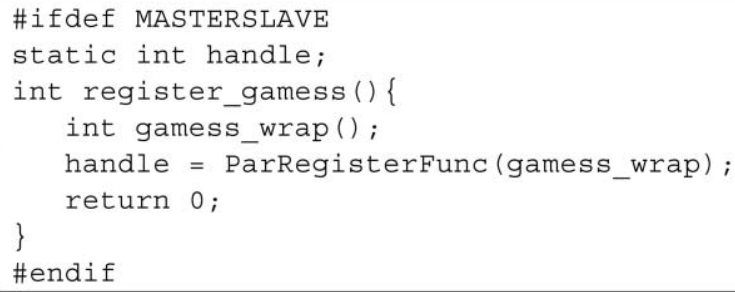

The resulting integer handle is then stored for future use.

The ChemShell input file (based on a Tcl script) is parsed by processor 0 and most commands are then executed in serial fashion, until a parallised module is invoked.
At this point, the ChemShell utility ParExecFunc() with the appropriate handle is invoked, causing a message to be sent to all the processors which, when received, will cause them all to call gamess_wrap(). In the serial case, gamess_wrap() is invoked directly.

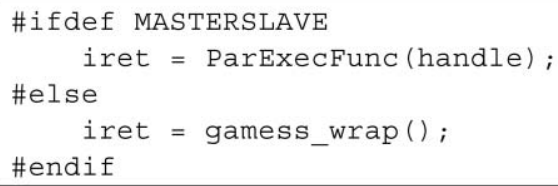

This mechanism allows ChemShell to comprise a mixture of serial and parallel code, with most I/O and housekeeping occurring on processor 0 .

Parallelism within the more loosely interfaced packages is handled differently, codes (such as TURBOMOLE) which run in parallel on workstation clusters can be run as stand-alone jobs, controlled by a serial ChemShell process. On the HPCx system, however, the cost of creating a parallel GAMESS-UK job for every geometry optimisation point would be substantial, due to the overheads associated with reading the binary on all nodes of the machine, and initialisation of the MPI message-passing framework on every node.

\section{Optimisation of the parallel code}

Here we will discuss some recent optimisation work by the HPCx tera-scaling team (P. Sherwood, I. J. Bush) in which a new version of ChemShell has been built. The work is based on recent developments to the GAMESS-UK code in which ScaLAPACK linear algebra tools have been used to implement a distributed data implementation with reduced I/O requirements. This has necessitated conversion of the parallel communication calls from the rest of the ChemShell from TCGMSG to MPI (a straightforward operation as the standard parallel ports of the relevant component codes, such as DL_POLY ${ }^{8}$ and GULP $1.3^{40}$ are based on MPI). The results are shown in Fig. 4, for a zeolite cluster similar to those

\section{QM/MM Geometry Optimisation Cycle Time}

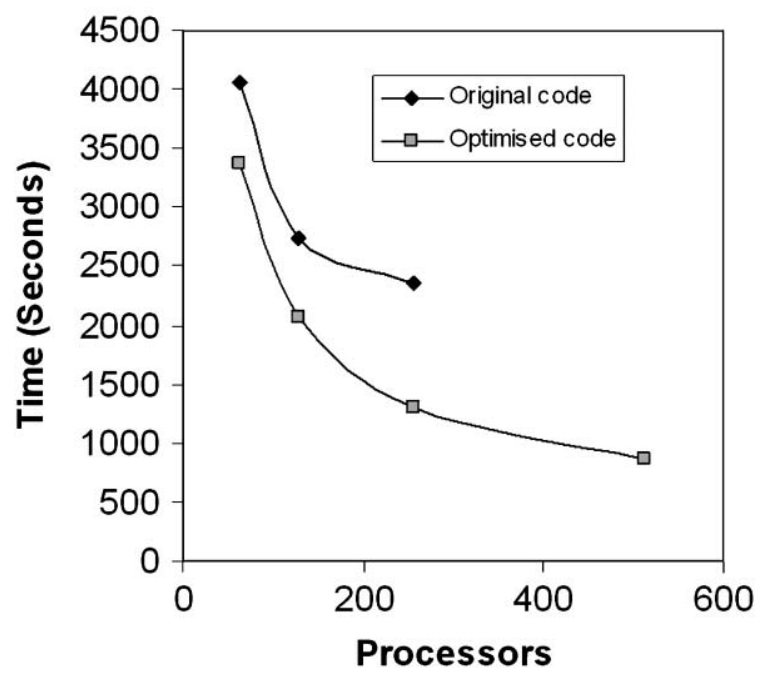

Fig. 4 Scalability of the HPCx implementation of ChemShell. 
used in the current paper but with a larger QM region (157 atoms, 1920 basis functions), where the cycle time as a function of processor count is shown for the original code.

The benefits arise from a variety of sources, but the main benefits are from the improved scalability of the underlying GAMESS-UK version (a better parallel diagonaliser, reduced I/O requirements etc.) and the use of MPI point-to-point communications rather than calls to the low-level communications API (LAPI) as used in the previous version.

\section{Choice of model clusters}

As neutron diffraction studies show that $\mathrm{Ti}$ preferentially occupies a T8 site in silicalite structure, ${ }^{41}$ we focus this work on the hydrolysis of a T8 site only. Six structurally distinct Ti clusters (many of which have been postulated in the literature) are chosen to model the Ti sites in TS-1, as shown in Fig. 5. In addition to the tetrapodal model (Fig. 5a), five possible combinations of the presence of $\mathrm{Ti}-\mathrm{OH}$ and/or $\mathrm{Si}-\mathrm{OH}$ groups have been studied. The first has three anchoring $\mathrm{Ti}-\mathrm{O}-\mathrm{Si}$ bridges, with the resulting silanol and titanol groups forming a two-membered ring (counting metal sites only). Thus, the structure which has a five coordinate Ti site will hereafter be referred to as $2 \mathrm{MR}$ (Fig. 5b). In the second model the Ti-OH group becomes inverted, which as a result breaks the twomembered ring and reforms the four coordinate $\mathrm{Ti}$ site. Further rearrangement of the structure gives rise to a hydrogen bond interaction between the non-inverted silanol group and a framework oxygen adjacent to Ti. This structure will be denoted as the tripodal ( $\mathrm{Si}-\mathrm{OH})$ species (Fig. 5c). The third structure is similar to tripodal $(\mathrm{Si}-\mathrm{OH})$, except that both the $\mathrm{Si}-\mathrm{OH}$ and $\mathrm{Ti}-\mathrm{OH}$ groups are inverted and are placed away from each other. The doubly inverted structure will be called the tripodal species (Fig. 5d). The fourth model has two anchoring $\mathrm{Ti}-\mathrm{O}-\mathrm{Si}$ bridges, with the resulting $\mathrm{Ti}-\mathrm{OH}$ and $\mathrm{Si}-\mathrm{OH}$ groups positioned away from each other, forming the bipodal species (Fig. 5e). Similarly the fifth structure has two anchoring $\mathrm{Ti}-\mathrm{O}-\mathrm{Si}$ bridges, however, a titanyl group $(\mathrm{Ti}=\mathrm{O})$ is also present forming a three coordinate Ti. This structure will be referred to as the titanyl species (Fig. 5f).

In all cases, the QM region consisted of the $\mathrm{T} 8$ site, atoms within three coordination shells of the T8 site (shell-3) and atoms of the connecting 5- and 10-membered rings (Fig. 6). Only atoms in shell-3 (including the T8 atom), atoms of the connecting 5-membered ring and water molecules, were treated with TZVP basis sets, while the remaining QM atoms, including the terminating $\mathrm{H}$ atoms, were described with $\mathrm{SV}$ basis sets. ${ }^{42,43}$ All atoms that lie within a $10 \AA$ radius of the T8 centre were optimised.

Our calculations using the BB1K functional provided a very accurate description of the electronic structure of localised and paramagnetic defects in silica materials. ${ }^{44}$ For this reason, we employed the $\mathrm{BB} 1 \mathrm{~K}$ exchange correlation functional ${ }^{45}$ as implemented within the GAMESS-UK code. ${ }^{7}$ The MM region consists of all atoms not treated explicitly in the QM region. Interactions in the MM region were dealt with using the aluminosilicate interatomic potentials of Hill and Sauer ${ }^{46}$ with modified charges. ${ }^{47}$

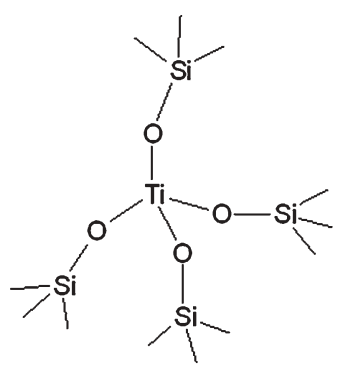

(a)

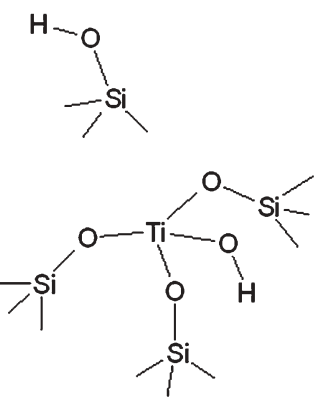

(d)

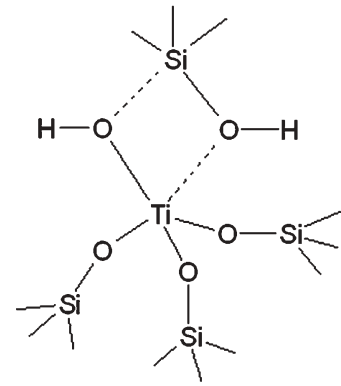

(b)

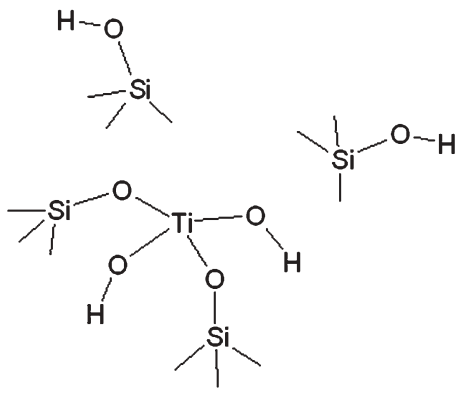

(e)

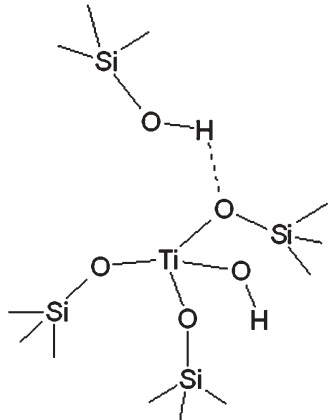

(c)

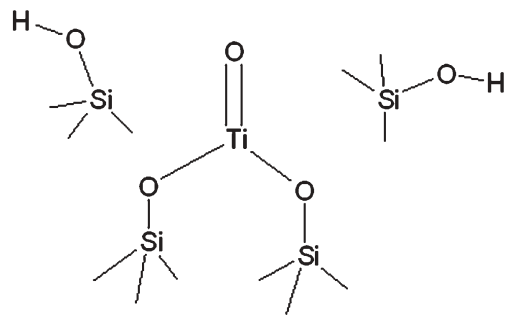

(f)

Fig. 5 The six models used to represent the active Ti sites in TS-1: (a) tetrapodal, (b) 2MR, (c) tripodal (Si-OH), (d) tripodal, (e) bipodal, and (f) titanyl. 


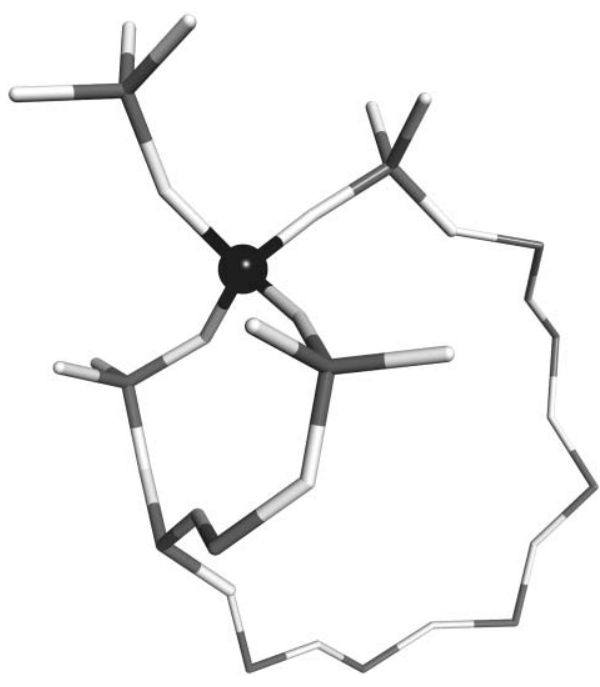

Fig. 6 A QM cluster with the tetrapodal model. The QM atoms treated with TZVP basis set are highlighted (sphere and cylinders), while the remaining atoms are described with $\mathrm{SV}$ basis sets. The $\mathrm{H}$ terminating atoms are omitted for clarity.

\section{QM/MM results for hydrolysis and inversion of tetrahedral $\mathrm{Ti}$ centres in TS-1}

\section{Reaction energies}

We found that the titanyl species requires the highest energy of formation among all structures studied $\left(142 \mathrm{~kJ} \mathrm{~mol}^{-1}\right.$ to form from the bipodal $\mathrm{Ti}$ by water extraction). As its presence in the catalyst is not supported by experiment either we will not consider it further.
The calculated energies of formation of the hydrolysed sites in silica and Ti-silicate are shown in the reaction scheme presented in Fig. 7.

The formation energy of the $\mathrm{Si}$ ion in the different structural environments indicates that the order of stability of these species decreases as: tripodal $>$ tetrapodal $>$ bipodal $>2 \mathrm{MR}$, whereas for the Ti silicate clusters, which includes the tripodal $(\mathrm{SiOH})$ intermediate, the order is slightly different: tripodal $>$ tripodal $(\mathrm{SiOH})>$ tetrapodal $>$ bipodal $>2 \mathrm{MR}$.

In both sets of calculations, the $2 \mathrm{MR}$ cluster is markedly higher in energy than the other three clusters, being 109 and $62 \mathrm{~kJ} \mathrm{~mol}^{-1}$ less stable than the pure silica and the Tisubstituted tetrapodal model, respectively. The large difference in energy is attributed to the formation of bridging silanol and/or titanol groups, which repel each other. However, the repulsion of these hydroxyl groups, which are analogous to bridging $\mathrm{Si}-\mathrm{O}(\mathrm{H})-\mathrm{Al}$ groups in acidic zeolites, is released after the inversion of the tetrahedral sites. During the inversion process, the $\mathrm{Si}$ and/or $\mathrm{Ti}$ atoms (bonded to the hydroxyl groups) move away from their tetrahedral configuration to a new 'external' relaxed position. The hydroxyl groups follow the T-atoms, which breaks the two-membered ring and forms the more stable tripodal structure.

Fig. 7 also shows that the tripodal structure is lower in energy by $12 \mathrm{~kJ} \mathrm{~mol}^{-1}$ than the tripodal $(\mathrm{SiOH})$. This result suggests that the silanol inversion process required to transform the tripodal $(\mathrm{SiOH})$ to the tripodal structure can easily occur under experimental conditions, and thus can be considered as an intermediate step in the formation of the tripodal structure. We can also conclude that the hydrogen bond formed between the silanol group and an adjacent bridging oxygen (with bond distance of $1.77 \AA$ ) in the tripodal

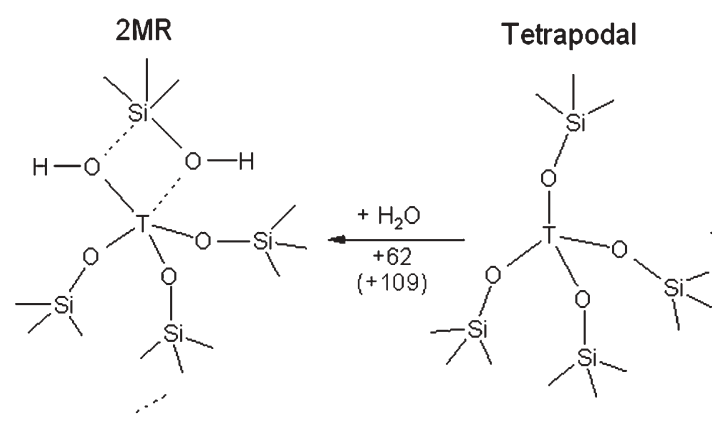

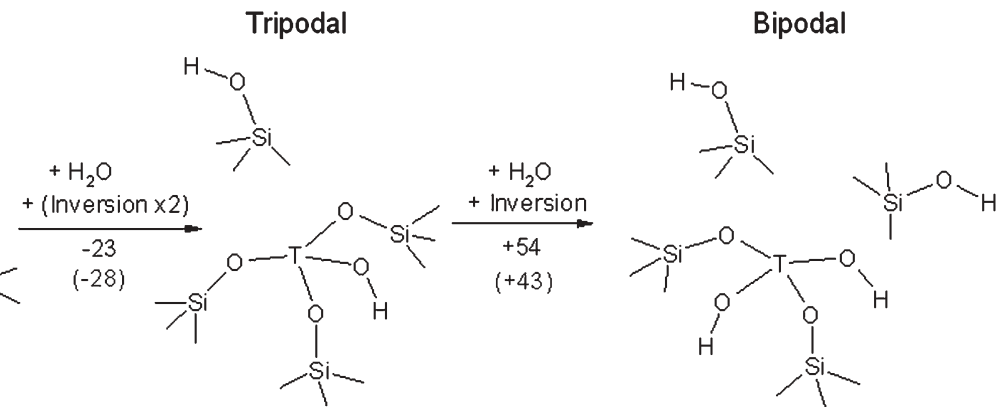

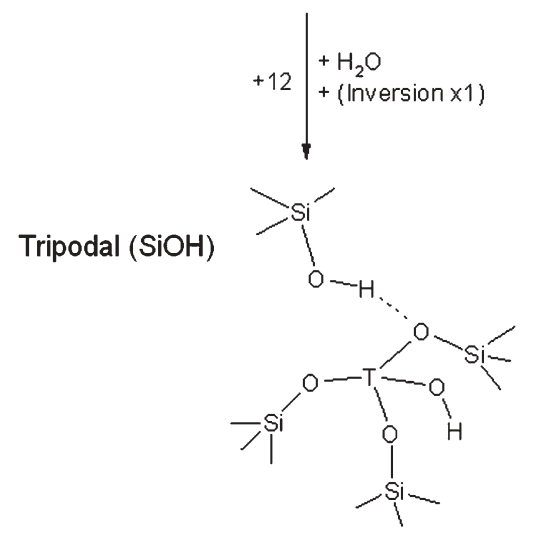

Fig. 7 Hydrolysis reaction scheme for Si and Ti sites in TS-1. Calculated formation energies in $\mathrm{kJ} \mathrm{mol}^{-1}$. The energies indicated are calculated from Ti- and Si-centred clusters ( $\mathrm{Si}$ values in parentheses). 
$(\mathrm{SiOH})$ structure is relatively weak, and thus can be easily broken. The weak character of the bond is also corroborated by a $119^{\circ}$ bond angle and a $0.97 \AA$ length of the hydroxyl bond, the values being in the characteristic range for free silanols.

It should also be noted that the formation of the tripodal site in the silicate host (via hydrolysis of the tetrapodal site) is calculated to be more exothermic (by $5 \mathrm{~kJ} \mathrm{~mol}^{-1}$ ) than the one formed in Ti substituted structures.

In summary, our results indicate that $\mathrm{T}$-atoms in tripodal structures are the most stable sites in anhydrous conditions, being $23 \mathrm{~kJ} \mathrm{~mol}^{-1}$ and $28 \mathrm{~kJ} \mathrm{~mol}^{-1}$ more energetically favourable than the tetrapodal $\mathrm{Ti}$ and $\mathrm{Si}$ sites, respectively. As already mentioned, the formation of the tripodal site involves the hydrolysis of one $\mathrm{T}-\mathrm{O}-\mathrm{Si}$ bridge, which as a result creates a silanol and a titanol group. In a real system, in the presence of water in zeolite channels, it is likely that these silanol and titanol groups are further stabilised by hydrogen bonds. Assuming that one of these groups is involved in only one hydrogen bond, and using the estimate of an H-bond strength as $\sim 20 \mathrm{~kJ} \mathrm{~mol}^{-1}$, there is little difference in energy between the tripodal and tetrapodal complexes. On the other hand, the formation of $2 \mathrm{MR}$ and bipodal sites in silica and Ti-silicate frameworks via hydrolysis of tetrapodal sites is calculated to be endothermic. Although the $2 \mathrm{MR}$ and bipodal sites are unstable with respect to both tetra- and tripodal sites, the hydrolysis reaction may be thermally assisted under experimental conditions. The only exception to this conclusion is the 2MR site in silicate, which is found to be highly unstable with respect to the tetrapodal site (by $109 \mathrm{~kJ} \mathrm{~mol}^{-1}$ ), and therefore would be present only as a short-lived intermediate. The tripodal, tripodal $(\mathrm{SiOH})$, tetrapodal, and to a lesser extent the bipodal and $2 \mathrm{MR}$ species are therefore proposed to be stable in the TS-1 framework, whilst only the tripodal, tetrapodal and bipodal sites are expected to be stable in pure silicalite.

\section{Structural features}

In this section we concentrate on the two ground state structures identified above. Tables 1 and 2 show a comparison of the geometrical parameters of the two structures with available EXAFS fitted data.

In line with EXAFS experiments, ${ }^{25}$ the main structural effect of Ti substitution is an increase in the $\mathrm{T}-\mathrm{O}$ bond lengths (by $0.14-0.18 \AA$ ), which is accompanied by an increase in the T-Si distances (by 0.08-0.17 $\AA$ ) and a small reduction in the

Table 1 Selection of calculated and experimental geometrical parameters of the Ti-silicate model of the tetrapodal T-sites. Bond distances in $\AA$ and bond angles in ${ }^{\circ}$

\begin{tabular}{|c|c|c|c|c|c|c|}
\hline Ti-O distance & Ti-S & distance & Ti-O & -Si angle & $\mathrm{O}-\mathrm{Ti}_{-}$ & -O angle \\
\hline Calc. Expt. & Calc. & Expt. $^{a}$ & Calc. & Expt. $^{a}$ & Calc. & Expt. $^{a}$ \\
\hline $1.81 \pm 0.02$ & 3.17 & $3.21 \pm 0.02$ & 139 & $141 \pm 5$ & 104 & 104-111 \\
\hline 1.78 & 3.27 & $3.25 \pm 0.02$ & 155 & $145 \pm 5$ & 105 & \\
\hline 1.79 & 3.35 & $3.37 \pm 0.02$ & 157 & $162 \pm 5$ & 108 & \\
\hline 1.80 & 3.37 & $3.37 \pm 0.02$ & 163 & $162 \pm 5$ & 110 & \\
\hline & & & & & 114 & \\
\hline & & & & & 115 & \\
\hline
\end{tabular}

Table 2 Selection of calculated and experimental geometrical parameters of the Ti-silicate model of the tripodal T-sites. Bond distances in $\AA$ and bond angles in ${ }^{\circ}$

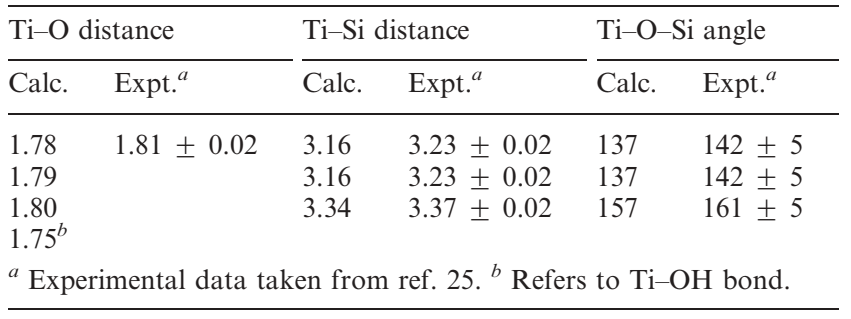

corresponding $\mathrm{T}-\mathrm{O}-\mathrm{Si}$ and $\mathrm{O}-\mathrm{T}-\mathrm{O}$ bond angles. The fact that the bond angles are only slightly distorted (less than $10^{\circ}$ ) suggests that the four $\mathrm{T}-\mathrm{O}-\mathrm{Si}$ and six $\mathrm{O}-\mathrm{Si}-\mathrm{O}$ angles are independent of the nature of the substituted cation, which is consistent with results from previous quantum mechanical calculations. $^{27,30}$

As reported from experiments on TS-1, the local geometry of the tetrapodal model has four Ti-O bond distances and four $\mathrm{Ti}-\mathrm{O}-\mathrm{Si}$ bond angles, which are not equivalent. ${ }^{25}$ If we combine closely related distances and their respective bond angles, we find that there are two average bond angles with values of 140 and $160^{\circ}$. EXAFS data on TS-1 also show similar values, however, the distribution of the four angles is different. In our calculated model, we find one $\mathrm{Ti}-\mathrm{O}-\mathrm{Si}$ bond angle at $140^{\circ}$ and three at $160^{\circ}$, whilst experiments predict two Ti-O-Si bond angles at $140^{\circ}$ and two at $160^{\circ}$. The splitting of the four $\mathrm{Ti}-\mathrm{O}-\mathrm{Si}$ angles into two couples was also obtained in several quantum mechanical studies. ${ }^{27,30}$ In Table 1 the experimental values refer to untreated TS-1 samples, however, additional results (not reported here) show that when TS-1 is pre-treated with ammonium acetate the distribution of the Ti-O-Si angle changes (one at $140^{\circ}$ and three at $160^{\circ}$ ). Although these bond angles of the pre-treated TS- 1 samples are consistent with the results obtained here, it has been documented that tripodal sites are the most dominant species in treated samples. Furthermore, the quality of the EXAFS fit of treated TS-1 is found to be poorer than that of the untreated samples ( $R$-factor $\dagger$ of 24 versus 15), which stresses the point that comparisons between calculated and experimental results should be dealt with carefully. In the case of the tripodal cluster, the calculated structural parameters compare well with those obtained from TS-1 samples pre-treated with ammonium acetate, in agreement with experiments. According to Table 2 the three Ti-OSi distances are consistent with the experimental values reported at $1.81 \AA$ with an experimental error of $\pm 0.02 \AA$. Despite the fact that two of the computed $\mathrm{Ti}-\mathrm{Si}$ distances (both at $3.16 \AA$ ) are shorter than those observed (3.23 $\AA$ with $\pm 0.02 \AA$ error), the distribution of the three Ti-Si distances, as well as the distribution of the $\mathrm{Ti}-\mathrm{O}-\mathrm{Si}$ angles for the calculated and experimental structures are in good agreement.

To our knowledge, there are no EXAFS data available for the bipodal model in TS-1. However, if we compare the

$\dagger R$-Factor is a measure of how close a theoretical model is to the experimental data: the lower the value the closer the fit. In ref. 37 the theoretical models were obtained from DFT cluster models. 
calculated data with those experimental results derived for tripodal models, the calculated $\mathrm{Ti}-\mathrm{O}$ distances are within the experimental uncertainty $( \pm 0.02 \AA)$, whereas the maximum value of the computed $\mathrm{Ti}-\mathrm{O}-\mathrm{Si}$ angle is significantly smaller than those found experimentally by $13-20^{\circ}$, which is clearly outside the experimental error $\left( \pm 5^{\circ}\right)$.

\section{Conclusions}

Based on these results we conclude that under anhydrous conditions a tetrapodal structure will probably constitute the ground state of the substitutional site. However, upon hydration, dramatic change should take place with the tripodal structure being prevalent and this tripodal structure will be the active site of the TS- 1 catalyst.

This study illustrates the type of complex chemical problem that can be tackled on a modern parallel computer such as the HPCx system. At the moment, it is reasonable to exploit 64-256 processor partitions of the machine and work is under way to extend the scalability further and also to provide access to more demanding quantum mechanical methods, such as highly correlated wavefunctions and treatments for excited states.

\section{Acknowledgements}

We gratefully acknowledge useful discussions with G. Sankar, N. R. Shiju, P. E. Sinclair, C. M. Barker, J. M. Thomas, A. M. Beal, G. Berlier, J. M. Garcés, and A. Kuperman. J. T. wishes to thank EPSRC for funding. Computational resources for this work have been provided by the HPCx service via our membership of the Materials Chemistry Consortium and funded by the grant GR/S 13422 .

\section{References}

1 I. W. C. E. Arends and R. A. Sheldon, Appl. Catal., A, 2001, 212, 175.

2 B. Notari, Adv. Catal., 1996, 41, 253.

3 J. To, A. A. Sokol, S. A. French, P. Sherwood, H. J. J. van Dam and C. R. A. Catlow, Angew. Chem., 2006, in press.

4 See www.chemshell.org, and reference 6.

5 P. Sherwood, A. H. de Vries, S. J. Collins, S. P. Greatbanks, N. A. Burton, M. A. Vincent and I. H. Hillier, Faraday Discuss., 1997, 106, 79.

6 P. Sherwood, A. H. de Vries, M. F. Guest, G. Schreckenbach, C. R. A. Catlow, S. A. French, A. A. Sokol, S. T. Bromley, W. Thiel, A. J. Turner, S. Billeter, F. Terstegen, S. Thiel, J. Kendrick, S. C. Rogers, J. Casci, M. Watson, F. King, E. Karlsen, M. Sjøvoll, A. Fahmi, A. Schäfer and Ch. Lennartz, J. Mol. Struct. (THEOCHEM), 2003, 632, 1.

7 M. F. Guest, J. M. H. Thomas, P. Sherwood, I. J. Bush and H. J. J. van Dam, Mol. Phys., 2005, 103, 719.

8 W. Smith and T. Forester, J. Mol. Graphics, 1996, 14, 136.

9 W. Smith, C. W. Yong and P. M. Rodger, Mol. Simul., 2002, 28, 385-471.

10 E. M. Flanigen, J. M. Bennett, R. W. Grose, J. P. Cohen, R. L. Patton, R. M. Kirchner and J. V. Smith, Nature, 1978, 271, 512.

11 S. Bordiga, S. Coluccia, C. Lamberti, L. Marchese, A. Zecchina, F. Boscherini, F. Buffa, F. Genoni, F. Leofanti, G. Petrini and G. Vlaic, J. Phys. Chem., 1994, 98, 4125.
12 R. Millini, E. Previdi Massara, G. Perego and G. Bellussi, J. Catal., 1992, 137, 497.

13 A. Zecchina, S. Bordiga, C. Lamberti, G. Ricchiardi, D. Scarano, G. Petrini, G. Leofanti and M. Mantegazza, Catal. Today, 1996, 32, 97.

14 S. Bordiga, F. Geobaldo, C. Lamberti, A. Zecchina, F. Boscherini, F. Genoni, G. Leofanti, G. Petrini, M. Padovan, S. Geremia and G. Vlaic, Nucl. Instrum. Methods Phys. Res., Sect. B, 1995, 97, 23.

15 G. Ricchardi, A. Damin, S. Bordiga, C. Lamberti, G. Spano, F. Rivetti and A. Zecchina, J. Am. Chem. Soc., 2001, 123, 11409.

16 A. S. Soult, D. D. Poore, E. I. Mayo and A. E. Stiegman, J. Phys. Chem. B, 2001, 105, 2687.

17 P. Ratnasamy, D. Srinivas and H. Knozinger, Adv. Catal., 2004, 48, 1 .

18 C. Lamberti, S. Bordiga, A. Zecchina, G. Artioli, G. Marra and G. Spano, J. Am. Chem. Soc., 2001, 123, 2204.

19 C. M. Zicovich-Wilson and A. Corma, J. Phys. Chem. B, 2000, 104, 4134.

20 M. R. Boccuti, K. M. Rao, A. Zecchina, G. Leofanti and G. Petrini, Stud. Surf. Sci. Catal., 1989, 48, 133.

21 T. Blasco, M. A. Camblor, A. Corma, P. Esteve, J. M. Guil, A. Martinez, J. A. Perdigón-Melon and S. J. Valencia, J. Phys. Chem. B, 1998, 102, 75.

22 S. L. Njo, H. van Koningsveld and B. van der Graaf, J. Phys. Chem. B, 1997, 101, 10065.

23 C. Lamberti, S. Bordiga, D. Arduino, A. Zecchina, F. Geobaldo, G. Petrini, A. Carati, F. Villain and G. Vlaic, J. Phys. Chem. B, 1998, 102, 6382.

24 P. E. Sinclair and C. R. A. Catlow, Chem. Commun., 1997, 1881.

25 D. Gleeson, G. Sankar, C. R. A. Catlow, J. M. Thomas, G. Spano, S. Bordiga, A. Zecchina and C. Lamberti, Phys. Chem. Chem. Phys., 2000, 2, 4812.

26 P. Sinclair, G. Sankar, C. R. A. Catlow, J. M. Thomas and T. Maschmeyer, J. Phys. Chem. B, 1997, 101, 4232.

27 C. M. Barker, PhD thesis, UCL, University of London, London, 2000.

28 S. Bordiga, A. Damin, F. Bonino, A. Zecchina, G. Spano, F. Rivetti, V. Bolis, C. Prestipino and C. Lamberti, J. Phys. Chem. B, 2002, 106, 9892.

29 C. M. Zicovich-Wilson, R. Dovesi and A. Corma, J. Phys. Chem. $B, 1999, \mathbf{1 0 3}, 988$.

30 G. Ricchiardi, A. de Man and J. Sauer, Phys. Chem. Chem. Phys., 2000, 2, 2195.

31 A. Damin, S. Bordiga and A. Zecchina, J. Chem. Phys., 2002, 117, 226.

32 E. Fois, A. Gamba and E. Spanò, J. Phys. Chem. B, 2004, 108, 154.

33 C. J. Brinker and G. W. Sherer, Sol-Gel Science: The Physics and Chemistry of Sol-Gel Processing, Academic Press Inc., New York, 1990.

34 J. K. Rudra and W. B. Fowler, Phys. Rev. B, 1987, 35, 8223.

35 K. C. Snyder and W. B. Fowler, Phys. Rev. B, 1993, 48, 13238.

36 A. A. Sokol, C. R. A. Catlow, J. M. Garcés and A. Kuperman, J. Phys. Chem., 1998, 102, 10647.

37 A. A. Sokol, C. R. A. Catlow, J. M. Garcés and A. Kuperman, J. Phys. Chem. B, 2002, 106, 6163.

38 D. Bakowies and W. Thiel, J. Phys. Chem., 1996, 100, 10580-94.

39 J. K. Ousterhout: Tcl and the Tk toolkit, Addison Wesley, Reading, MA, 1994.

40 J. D. Gale, J. Chem. Soc., Faraday Trans., 1997, 93, 629-37.

41 P. F. Henry, M. T. Weller and C. C. Wilson, J. Phys. Chem. B, 2001, 105, 7452.

42 A. Schäfer, H. Horn and R. Ahlrichs, J. Chem. Phys., 1992, 97, 2571.

43 A. Schäfer, C. Huber and R. Ahlrichs, J. Chem. Phys., 1994, 100, 5829.

44 J. To, A. A. Sokol, S. A. French, N. Kaltsoyannis and C. R. A. Catlow, J. Chem. Phys., 2005, 122, 144704.

45 Y. Zhao and B. J. Lynch, J. Phys. Chem. A, 2004, 108, 6908.

46 J. R. Hill and J. Sauer, J. Phys. Chem., 1995, 99, 9536.

47 A. H. de Vries, P. Sherwood, S. J. Collins, A. M. Rigby, M. Rigutto and G. J. Kramer, J. Phys. Chem. B, 1999, 103, 6133. 